NASA Contractor Report 179556

AIAA-87-0600

\title{
An LU-SSOR Scheme for the Euler and Navier-Stokes Equations
}

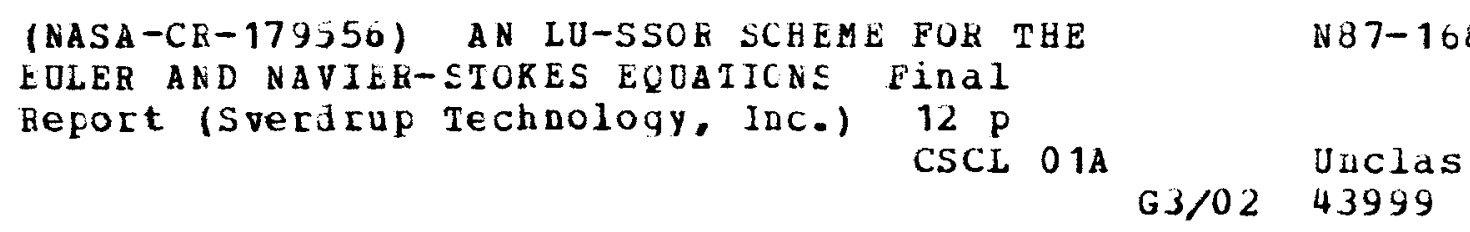

Seokkwan Yoon

Sverdrup Technology, Inc.

Lewis Research Center

Cleveland, Ohio

and

Antony Jameson

Princeton University

Princeton, New Jersey

December 1986

Prepared for

Lewis Research Center

Under Contract NAS3-24105

\section{N/Sก}

National Aeronautics and

Space Administration 


\author{
Seokkwan Yoon* \\ Sverdrup Technology, Inc. \\ Lewis Research Center \\ Cleveland, Ohio 44135 \\ and \\ Antony Jameson** \\ Princeton University \\ Princeton, New Jersey 08544
}

\section{Abstract}

A new multigrid relaxation scheme, lower-upper symmetric successive overrelaxation (LU-SSOR) scheme, is developed for the steady-state solution of the Euler and Navier-Stokes equations. The scheme, which is based on central differences, does not require flux splitting for approximate Newton iteration. Application to transonic flow shows that the new method is efficient and robust. The vectorizable LU-SSOR scheme needs only SCalar diagonal inversions.

\section{Introduction}

The Reynolds numbers for a larqe airplane are of the order of thirty million. Therefore, laminar flow in the boundary layer becomes unstable, resulting in turbulent flow over most of the surface of the airplane. However, the computational requirements for the simulation of turbulent flow are clearly beyond the reach of current computers. The first level of approximation - time averaging the rapidly fluctuating components - yields the Reynolds-averaged Navier-Stokes equations, which require a turbulence model for closure. At the present time not much is known about the behavior of turbulence in separated regions, and this has impeded the development of turbulence models for complex three-dimensional flows. Since a universally satisfactory turbulence model has yet to be found, current turbulence models have to be tailored to the particular flow. During the last decade the feasibility of solving the Navier-Stokes equations has been explored but the methods developed so far have been too expensive to use in a routine production mode.

Recently several implicit schemes have been developed successfully in conjunction with a multigrid method for steady-state solution of the unsteady Euler equations. 1,2 Although the alternating-direction implicit scheme could be improved to achieve the expected efficiency of the multigrid method in two dimensions, 1 its inherent 1 imitations in three dimensions suggest alternative approaches. 2 An alternative implicit scheme that is stable in any number of space dimensions is based on lower-upper (LU) factorization. The LU implicit scheme proved to be robust and efficient for highspeed flows up to Mach 20 as well as for transonic flows. $3-5$ It was also shown that a symmetric

* Senior Engineer, Sverdrup Technology, Inc. Member AIAA

$\star \star$ Professor, Department of Mechanical and Aerospace Engineering Member AIAA
Gauss-Seidel relaxation method for solving the unfactored implicit scheme was a variant of the LU implicit scheme.

The Newton iteration method has been investigated to solye the steady Euler or Navier-Stokes equations.6-8 Because of the rapid growth of the operation count with the number of mesh cells, the system was solved indirectly. Jespersen 6 and Hemker and Spekreijse applied the symmetric

Gauss-Seidel method to the Euler equations, while MacCormack ${ }^{8}$ applied the line Gauss-Seidel method to the Navier-Stokes equations. In this paper an efficient multigrid relaxation scheme is developed for approximate Newton iteration. The new lowerupper symmetric success ive overrelaxation (LU-SSOR) scheme requires scalar diagonal inversions while the Gauss-Seidel method and the LU implicit scheme require block matrix inversions. The use of scalar diagonal inversions offers the potential for orderof-magnitude speedups when large systems of partial differential equations must be solved, for example, for hypersonic flows with finite-rate chemistry. It is desirable that the matrix be diagonally dominant to assure the convergence of a relaxation method. The new method based on central differences achieves this without the flux splitting that substantially increases the computational work per cycle. Numerical examples include inviscid and viscous transonic airfoils.

\section{Governing Equations}

The Navier-Stokes equations represent gas flow in thermodynamic equilibrium. Let $t, \rho, E, T$, and $p$ be time, density, total energy, temperature, and pressure; $u$ and $v$ Cartesian velocity components; and $x$ and $y$ Cartesian coordinates. Then for a two-dimensional flow these equations can be written as

$$
\frac{\partial W}{\partial t}+\frac{\partial F}{\partial x}+\frac{\partial G}{\partial y}=\frac{\partial F v}{\partial x}+\frac{\partial G_{v}}{\partial y}
$$

where $W$ is the vector of dependent variables, and $F$ and $G$ are convective flux vectors:

$$
\left.\begin{array}{c}
W=(\rho, \rho u, \rho v, \rho E)^{\star} \\
F=\left[\rho u, \rho u^{2}+\rho, \rho v u, u(\rho E+p)\right]^{\star} \\
G=\left[\rho v, \rho u v, \rho v^{2}+p, v(\rho E+p)\right]^{\star}
\end{array}\right\}
$$

Here * denotes the transpose of a matrix. The flux vectors for the viscous terms are

$$
F_{v}=\left(0, \tau_{x x}, \tau_{x y}, u \tau_{x x}+v \tau_{x y}+k \frac{\partial T}{\partial x}\right) \text { * }
$$




$$
G_{v}=\left(0, \tau_{x y}, \tau_{y y}, u \tau_{x y}+v_{\tau_{y y}}+k \frac{\partial T}{\partial y}\right) *
$$

Here the viscous stresses are

$$
\begin{gathered}
\tau_{x x}=2 \mu u_{x}-\frac{2}{3} \mu\left(u_{x}+v_{y}\right) \\
\tau_{x y}=\mu\left(u_{y}+v_{x}\right)
\end{gathered}
$$

and

$$
\tau_{y y}=2 u v_{y}-\frac{2}{3} u\left(u_{x}+v_{y}\right)
$$

where $\mu$ is the coefficient of viscosity and $k$ is the coefficient of thermal conductivity. state:

The pressure is obtained from the equation of

$$
p=\rho(r-1)\left\{E-\frac{1}{2}\left(u^{2}+v^{2}\right)\right\}
$$

where $r$ is the ratio of specific heats.

\section{Semi-Discrete Finite-Volume Method}

A convenient way to assure a steady-state solution independent of the time step is to separate the space and time discretization procedures. In the semi-discrete finite-volume method one begins by applying a semi-discretization in which only the spatial derivatives are approximated. Using a finite-volume method for space discretization allows one to handle arbitrary geometries and helps to a void problems with the metric singularities that are usually associated with finite-difference methods. Finite-volume methods do not require special treatment on a composite grid. ${ }^{9}$ The scheme reduces to a central difference scheme on a Cartesian grid and is second-order accurate in space provided the mesh is smooth enough, and uniform flow is an exact solution of the difference equations. The Gauss theorem is used to evaluate the viscous flux terms. 10

\section{Nonlinear Adaptive Dissipation}

Using a central difference scheme when calculating flows with discontinuities typically produces flowfield oscillations in the neighborhood of shock waves, where the pressure gradients are severe. To suppress the tendency for spurious odd and even point oscillations and to prevent nonphysical overshoots near shock waves, we augment the scheme by artificial dissipative terms. The dissipative term, which is constructed so that it is third-order accurate in smooth regions of the flow, is explicitly added to the residual. For the density equation, for example, the dissipation $d$ has the form

$$
d_{j+1 / 2, j}-d_{i-1 / 2, j}+d_{i, j+1 / 2}-d_{i, j-1 / 2}
$$

where

$$
\begin{aligned}
& d_{i+1 / 2, j}=\varepsilon_{i+1 / 2, j}^{(2)}\left(\rho_{i+1, j}-\rho_{i, j}\right) \\
& -\varepsilon_{i+1 / 2, j}^{(4)}\left(\rho_{i+2, j}-3 \rho_{i+1, j}+3 \rho_{i, j}-\rho_{i-1, j}\right)
\end{aligned}
$$

Here $\varepsilon_{\varepsilon}(2)$ and $\varepsilon^{(4)}$ denote coefficients of the second and fourth differences respectively. The cell area $S$ is equivalent to the inverse of the transformation Jacobian determinant. Both coefficients include a normalizing factor, $S_{j}+1 / 2, j / \Delta t$, proportional to the length of the cell side, and $\varepsilon_{i+1 / 2, j}^{(2)}$ is also made proportional to the normalized second difference of the pressure:

$$
v_{i, j}=\left|\frac{p_{i+1, j}-2 p_{i, j}+p_{i-1, j}}{p_{i+1, j}+2 p_{i, j}+p_{i-1, j}}\right|
$$

in the adjacent cells. The third-order terms provide background damping of high-frequency modes. The first-order terms, which are needed to control oscillations in the neighborhood of shock waves, are activated when strong pressure gradients in the flow are sensed. The dissipative terms for the other equations are constructed from similar formulas. Increasing the amount of artificial viscosity improves the rate of convergence, although too much dissipation can hinder this. However, dissipation should be as small as possible so that the accuracy of the solution is not degraded. The typical amount of the third-order terms is almost negligible when compared to the physical viscosity. Schemes constructed along these lines combine the advantages of simplicity and economy of computation. They have also proved robust in calculations over a wide range of Mach numbers. 3 For more accurate capturing of oblique shock waves in hypersonic flows, a total variation diminishing (TVD) scheme ${ }^{4}$ can be used.

\section{LU-SSOR Scheme}

A prototype implicit scheme for a system of nonlinear hyperbolic equations such as the Euler equations can be formulated as

$$
\begin{aligned}
W^{n+1}=W^{n}-\beta \Delta t & {\left[D_{x} F\left(W^{n+1}\right)+D_{y} G\left(W^{n+1}\right)\right] } \\
& -(1-\beta) \Delta t\left[D_{x} F\left(W^{n}\right)+D_{y} G\left(W^{n}\right)\right]
\end{aligned}
$$

where $D_{x}$ and $D_{y}$ are central difference operators that approximate a/ax and a/ay. Here $n$ denotes the time level. An enormous number of computations must be performed when the scheme is in this form because coupled nonlinear equations must be solved at each time step. Let the Jacobian matrices be

$$
\left.\begin{array}{l}
A=\frac{\partial F}{\partial W} \\
B=\frac{\partial G}{\partial W}
\end{array}\right\}
$$

and let the correction be

$$
\delta W=W^{n+1}-W^{n}
$$

The scheme can be linearized by setting

$$
\begin{aligned}
& F\left(W^{n+1}\right)=F\left(W^{n}\right)+A \delta W+0\left(\|\delta W\|^{2}\right) \\
& G\left(W^{n+1}\right)=G\left(W^{n}\right)+B \delta W+O\left(\|\delta W\|^{2}\right)
\end{aligned}
$$

where 0 is the order of the enclosed terms, and dropping terms of the second and higher orders to yield 


$$
\left[I+\beta \Delta t\left(D_{x} A+D_{y} B\right)\right] \delta W+\Delta t R=0
$$

where $I$ is the identity matrix and $R$ is the residual

$$
R=D_{x} F\left(W^{n}\right)+D_{y} G\left(W^{n}\right)
$$

If a constant $\beta=1 / 2$, the scheme remains secondorder accurate in time; for other values of $B$, the time accuracy drops to first order.

The unfactored implicit scheme (Eq. (8)) produces a large block banded matrix that can be inverted only by performing a great many computations. In addition, a large amount of storage is required. If $B=1$ the scheme reduces to a Newton iteration in the limit $\Delta t \rightarrow \infty$ :

$$
\left(D_{x} A+D_{y} B\right) \delta W+R=0
$$

A diagonally dominant form of Eq. (9)

$$
\left(D_{x}^{-} A^{+}+D_{x}^{+} A^{-}+D_{y}^{-} B^{+}+D_{y}^{+} B^{-}\right) \delta W+R=0
$$

can be written as

$$
\begin{aligned}
A_{i j}^{+} \delta W_{i j} & -A_{i-1, j}^{+} \delta W_{i-1, j}+A_{i+1, j}^{-} \delta W_{i+1, j} \\
& -A_{i j}^{-} \delta W_{i j}+B_{i j}^{+} \delta W_{i j}-B_{i, j-1}^{+} \delta W_{i, j-1} \\
& +B_{i, j+1}^{-} \delta W_{i, j+1}-B_{i j}^{-} \delta W_{i j}+R_{i j}=0
\end{aligned}
$$

By simulating it with backward and forward relaxation sweeps, we obtain the symmetric successive overrelaxation (SSOR) method, which can be written in two steps as

$$
\begin{aligned}
\left(A_{i j}^{+}-A_{i j}^{-}\right) \delta W_{i j}^{*}+ & A_{i+1, j}^{-} \delta W_{i+1, j}^{\star}+\left(B_{i, j}^{+}-B_{i j}^{-}\right) \delta W_{i j}^{\star} \\
& +B_{i, j+1}^{-} \delta W_{i, j+1}^{\star}+R_{i j}=0
\end{aligned}
$$

followed by

$$
\begin{aligned}
\left(A_{i j}^{+}-A_{i j}^{-}\right) \delta W_{i j} & -A_{i-1, j}^{+} \delta W_{i-1, j}^{+} A_{i+1, j}^{-} \delta W_{i+1, j}^{*} \\
& +\left(B_{i j}^{+}-B_{i j}^{-}\right) \delta W_{i j}-B_{i, j-1}^{+} \delta W_{i, j-1} \\
& +B_{i, j+1}^{-} \delta W_{i, j+1}^{*}+R_{i j}=0
\end{aligned}
$$

where $D_{x}^{-}$and $D_{y}^{-}$are backward difference operators and ${D_{x}^{+}}_{x}$ and ${D_{y}^{+}}_{\text {are forward difference }}^{+}$ operators. Here, two-point operators are used for steady flow calculations. $A^{+}, A^{-}, B^{+}$, and $B^{-}$are constructed so that the eigenvalues of "+" matrices are nonnegative and those of "-" matrices are nonpositive:

$$
\begin{aligned}
& A^{+}=\frac{1}{2}\left(A+r_{A} I\right) \\
& A^{-}=\frac{1}{2}\left(A-r_{A} I\right) \\
& B^{+}=\frac{1}{2}\left(B+r_{B} I\right) \\
& B^{-}=\frac{1}{2}\left(B-r_{B} I\right)
\end{aligned}
$$

where

$$
\left.\begin{array}{l}
r_{A} \geq \max \left(\left|\lambda_{A}\right|\right) \\
r_{B} \geq \max \left(\left|\lambda_{B}\right|\right)
\end{array}\right\}
$$

Here, $\lambda_{A}$ and $\lambda_{B}$ represent eigenvalues of Jacobian matrices.

Subtract Eq. (12) from Eq. (13) to get

$$
\begin{aligned}
& \left(A_{i j}^{+}-A_{i j}^{-}\right) \delta W_{i j}-A_{i-1, j}^{+} \delta W_{i-1, j}+\left(B_{i j}^{+}-B_{i j}^{-}\right) \delta W_{i j} \\
& -B_{i, j-1}^{+} \delta W_{i, j-1}=\left(A_{i j}^{+}-A_{i j}^{-}\right) \delta W_{i j}^{\star}+\left(B_{i j}^{+}-B_{i j}^{-}\right) \delta W_{i j}^{*}
\end{aligned}
$$

This may be written as

$$
\left(D_{x}^{-} A^{+}+D_{y}^{-} B^{+}-A^{-}-B^{-}\right) \delta W=\left(A^{+}+B^{+}-A^{-}-B^{-}\right) \delta W^{\star}
$$

where

$$
\delta W^{*}=\left(D_{x}^{+} A^{-}+D_{y}^{+} B^{-}+A^{+}+B^{+}\right)^{-1}(-R)
$$

If we take "+" and "-" matrices as given in

Eq. (14),

$$
\begin{aligned}
& A^{+}-A^{-}=r_{A} I \\
& B^{+}-B^{-}=r_{B} I
\end{aligned}
$$

Thus, Eq. (17) becomes the LU-SSOR scheme for approximate Newton iteration

$$
\begin{aligned}
\left(D_{x}^{-} A^{+}\right. & \left.+D_{y}^{-} B^{+}-A^{-}-B^{-}\right)\left(D_{x}^{+} A^{-}+D_{y}^{+} B^{-}\right. \\
& \left.+A^{+}+B^{+}\right) \delta W=-\left(r_{A}+r_{B}\right)\left(D_{x} F+D_{y} G\right)
\end{aligned}
$$

which can be inverted in two steps.

For the Navier-Stokes equations, $F$ and $G$ are replaced by $F-F_{V}$ and $G-G_{V}$ in Eq. (19). That is, 


$$
\begin{aligned}
\left(D_{x}^{-} A^{+}\right. & \left.+D_{y}^{-} B^{+}-A^{-}-B^{-}\right)\left(D_{x}^{+} A^{-}+D_{y}^{+} B^{-}+A^{+}+B^{+}\right) \delta W \\
& =-\left(r_{A}+r_{B}\right)\left[D_{x}\left(F-F_{v}\right)+D_{y}\left(G-G_{v}\right)\right. \\
& \left.-\varepsilon_{x}^{(2)} D_{x}^{2} W+\varepsilon_{x}^{(4)} D_{x}^{4} W-\varepsilon_{y}^{(2)} D_{y}^{2} W+\varepsilon_{y}^{(4)} D_{y}^{4} W\right]
\end{aligned}
$$

where $D^{2}$ and $D^{4}$ denote the second difference and the fourth difference operators respectively.

Since one-sided difference schemes are naturally dissipative, no implicit smoothing is required on the left side. Only adaptive dissipation terms are explicitly added to the residual on the right side. It is interesting to note that the present numerical method eliminates the need for block diagonal inversions without using the diagonalization process. This is an especially desirable feature for the analysis of hypersonic reacting flows. The LU family of algorithms are fully vectorizable along $i+j=$ constant 1 ines on a vector computer.

\section{Multigrid Method}

The underlying idea of a multigrid method is to transfer some of the task of tracking the evolution of the system to a sequence of successively coarser meshes. 11 This has two advantages.

First, the computational effort per cycle is reduced on a coarser mesh. Second, the use of larger control volumes on the coarser grids tracks the evolu$t$ ion on a larger scale, with the consequence that global equilibrium can be more rapidly attained. In general one can conceive of a multigrid method using a sequence of independently generated coarser meshes which are not associated with each other in any structured way. Here attention is restricted to the case in which coarser meshes are generated by eliminating alternating points in each coordinate direction. Thus simple rules can be formulated for the transfer of data between grids. The cells of the fine mesh can be amalgamated into larger cells which form a coarser mesh. Then in each coarse mesh cell the conservation laws are represented by summing the flux balances of its fine mesh cells; consequently, the evolution on the coarse mesh is driven by the disequilibrium of the fine mesh equations. The multigrid method used here is the cellcentered method which was used for the implicit schemes.1,2

\section{Results}

The first test case was for inviscid transonic flow past the NACA 0012 airfoil at $1.25^{\circ}$ angle of attack. The freestream Mach number was 0.8 . Nonreflecting boundary conditions were used to absorb the waves impinging on the far-field boundary.? Figure 1 shows the plot of Mach number contours. Convergence histories of the LU-SSOR scheme are compared with those of the LU implicit scheme of Ref. 2. Figure 2 shows the logarithm of the average density residuals, and $\mathrm{Fig}$. 3 shows the 1 ift histories. Five-level multigrid calculations were performed on a 128 by 32 C-mesh without grid sequencing. Uniform flow was given as the initial condition. As the results show, the convergence rate of the LU-SSOR scheme is about 30 percent faster than that of the LU imp'icit scheme. Moreover, the computational work per cycle for the
LU-SSOR scheme is about 30 percent less than that for the LU implicit scheme since the LU-SSOR scheme does not need block diagonal inversion. This offers the potential for order-of-magnitude speedups when large systems of partial differential equations must be solved. For example, the size of Jacobian matrices for scramjet flows can be over 20 by 20 in three dimensions if 15 species equations are added. It seems that slow convergence of the LU-SSOR implicit scheme in Ref. 2 is due to the method of local time stepping that was used in Ref. 2 .

The next case was for viscous laminar flow past the NACA 0012 airfoil at Mach 0.5, Reynolds number 5000 , and zero angle of attack. The adjabatic wall boundary condition was used at the body surface. Calculations were performed on a stretched 192 by $48 \mathrm{C}$-mesh. A convergence acceleration technique such as enthalpy damping was not used for the viscous flow calculations. Figure 4 shows the Mach number contours while Fig. 5 shows velocity vectors. Convergence history with a two-level multigrid is shown in Fig. 6. Theoretically, improved convergence rates might be obtained by using more levels of multigrid.

The last case was for viscous turbulent flow past the RAE 2822 airfoil at Mach 0.73 , Reynolds number 6.5 million, and $2.79^{\circ}$ angle of attack. The Reynolds-averaged Navier-Stokes equations were, solved using a Baldwin-Lomax turbulence model.12 Transition was fixed at 3 percent chord. Mach number contours are shown in Fig. 7 (the dashed line denotes the sonic line). Convergence histories on a 192 by 48 mesh with two-level multigrid are shown: $\mathrm{Fig}$. 8 shows the residual history and Fig. 9 shows the lift and drag history. The convergence based on the average residual is continuous although it is slowed after dropping about three orders of magnitude. It jeems possible to improve the accuracy and the convergence rate further by using a better distribution of grid points.

\section{Conclusion}

A new relaxation scheme derived for approximate Newton iteration is applied to both Euler and Navier-Stokes equations. The LU-SSOR scheme is not only stable in three dimensions but also fully vectorizable. Transonic airfoil calculations confirm that the scheme is robust and efficient. Moreover, the LU-SSOR scheme is promising for high-speed reacting flow calculations.

\section{References}

1. Jameson, A. and Yoon, S., "Multigrid Solution of the Euler Equations Using Implicit Schemes," AIAA Journal, Vol. 24, No. 11, Nov. 1986, pp. 1737-1743.

2. Jameson, A. and Yoon, S., "LU Implicit Schemes with Multiple Grids for the Euler Equations," AIAA Paper 86-0105, Jan. 1986.

3. Yoon, S. and Jameson, A., "An LU Implicit Scheme for High Speed Inlet Analysis," AIAA Paper 86-1520, June 1986.

4. Yoon, S. and Jameson, A. "A High Resolution Shock Capturing Scheme for High Mach Number Internal Flow," NASA CR-179523, 1986. 
5. Sockol, P.M. and Yoon, S., "Advanced Numerical Methods for Hypersonic Internal Flows," The 2nd National Aerospaceplane Symposium, Applied Physics Lab., Johns Hopkins University, Baltimore, MD, Nov. 1986.

6. Jespersen, D.C., "A Multigrid Method for the Euler Equations," AIAA Paper 83-0124, Jan. 1983.

7. Hemker, P.W. and Spekreijse, S.P., "Multiple Grid and Osher's Scheme for the Efficient Solution of the Steady Euler Equations," Journal of Numerical Mathematics, Dec. 1986.

8. MacCormack, R.W., "Current Status of Numerical Solutions of the Navier-Stokes Equations," AIAA Paper 85-0032, Jan. 1985.
9. Choo, Y.K., Yoon, S., and Reno, C., "Generation of a Composite Grid for Turbine Flows and Consideration of a Numerical Scheme," NASA TM-88890, 1986.

10. Martinelli, L., Jameson, A., and Grasso, F., "A Multigrid Method for the Navier-Stokes Equations," AIAA Paper 86-0208, Jan. 1986.

11. Jameson, A., "Multigrid Algorithms for Compressible Flow Calculations," MAE Report 1743, Princeton University, Oct. 1985.

12. Baldwin, B.S. and Lomax, H., "Thin Layer Approximation and Algebraic Model for Separated Turbulent Flows," AIAA Paper 78-257, Jan. 1978. 


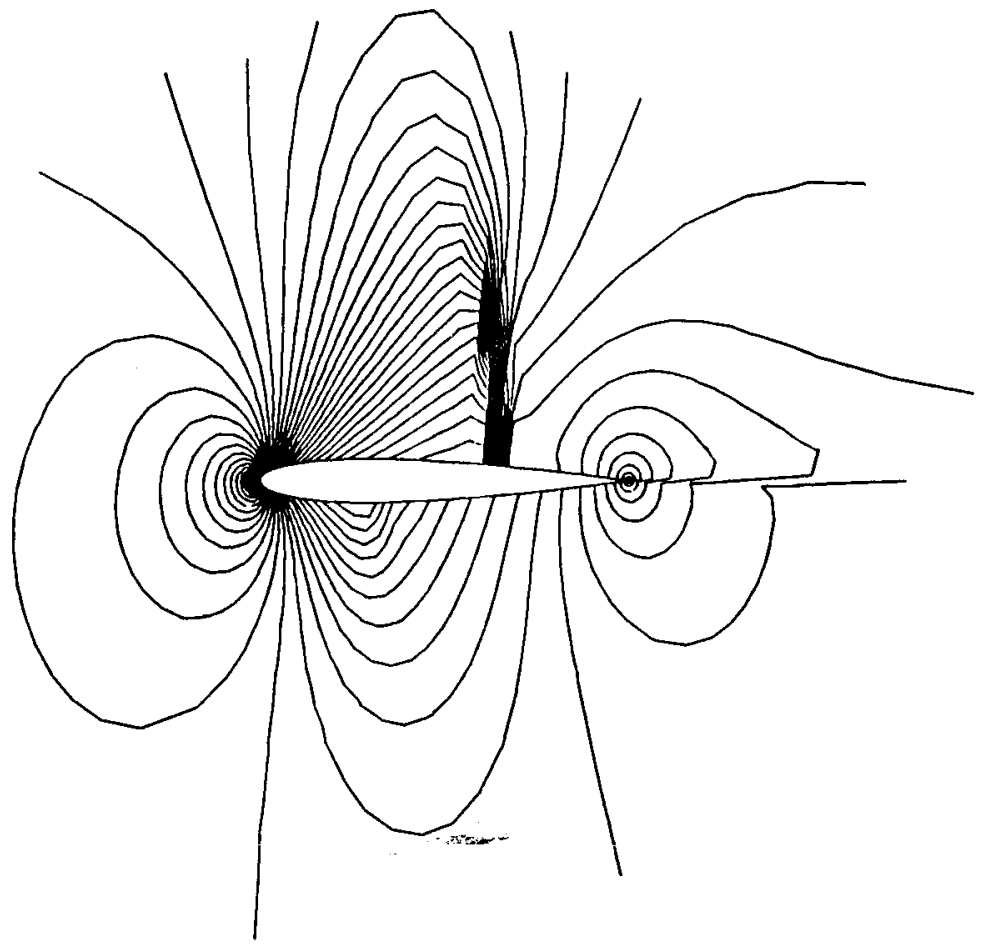

Figure 1. - Mach NUMBer contours fOR INVISCid tRanSONIC FLOW.

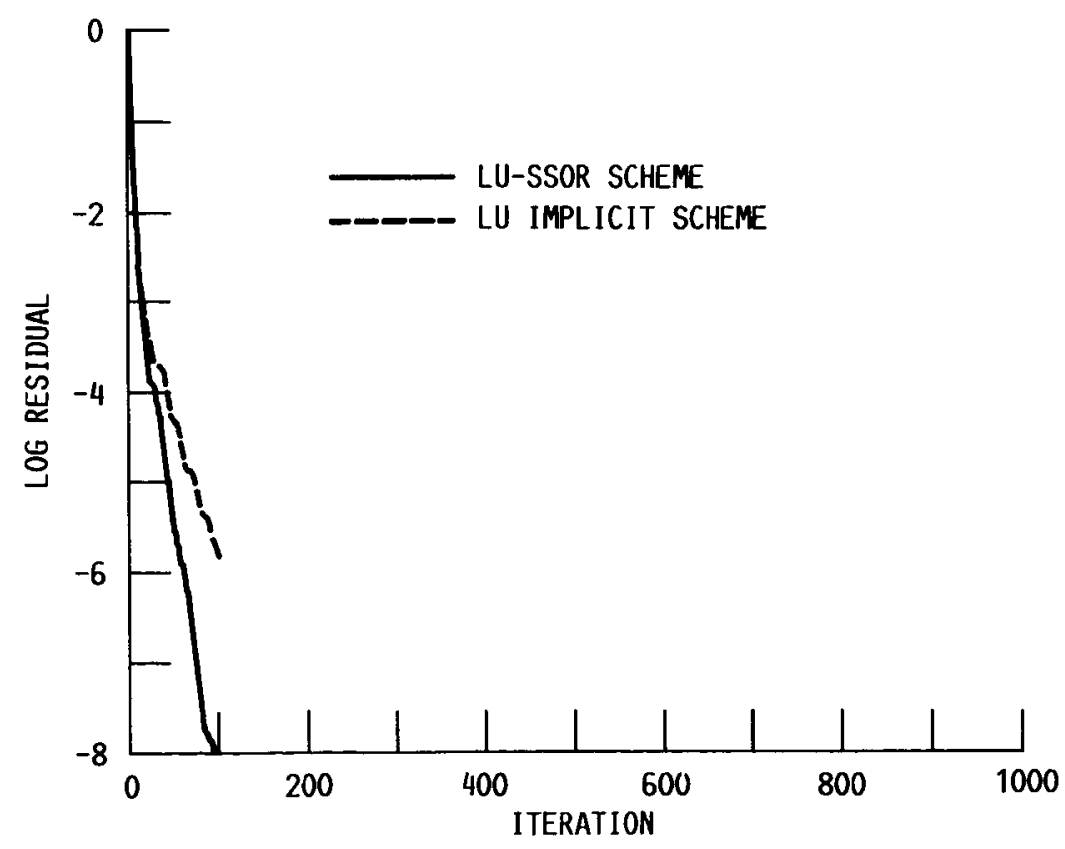

FigURE 2.- CONVERGENCE HISTORY FOR INVISCID TRANSONIC FLOW. 


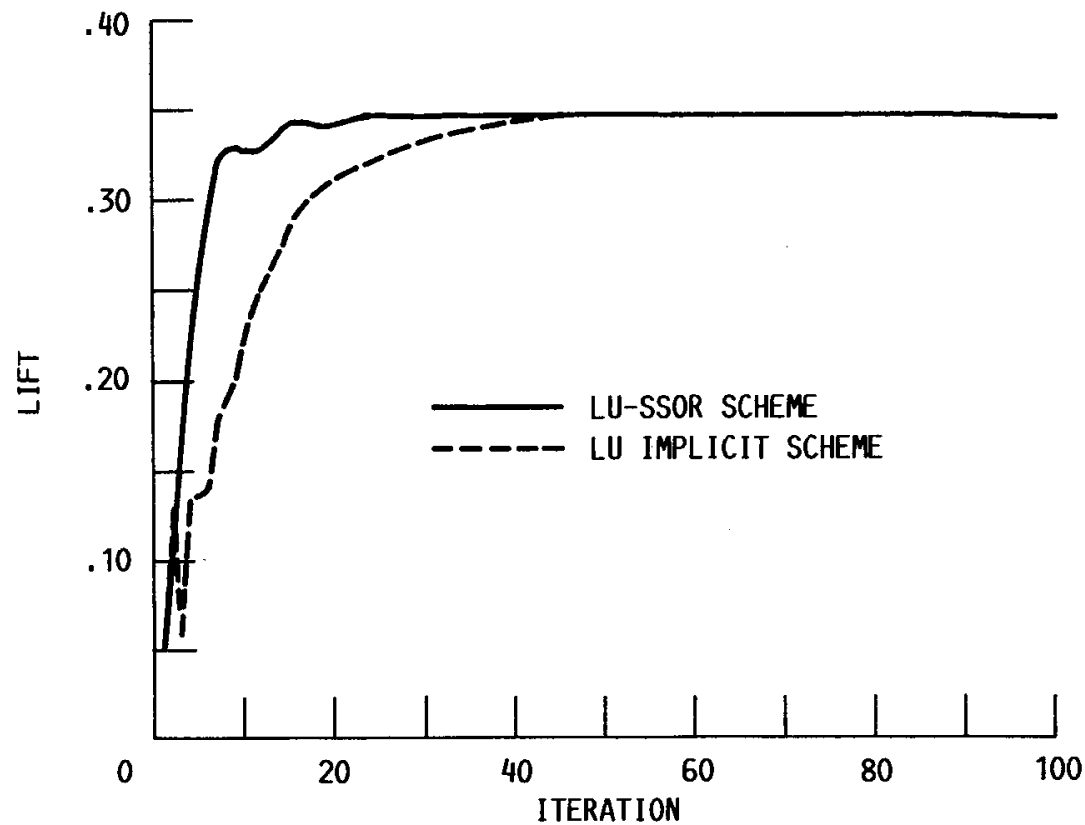

FIGURE 3.- LIFT HISTORY FOR INVISCID TRANSONIC FLOW.

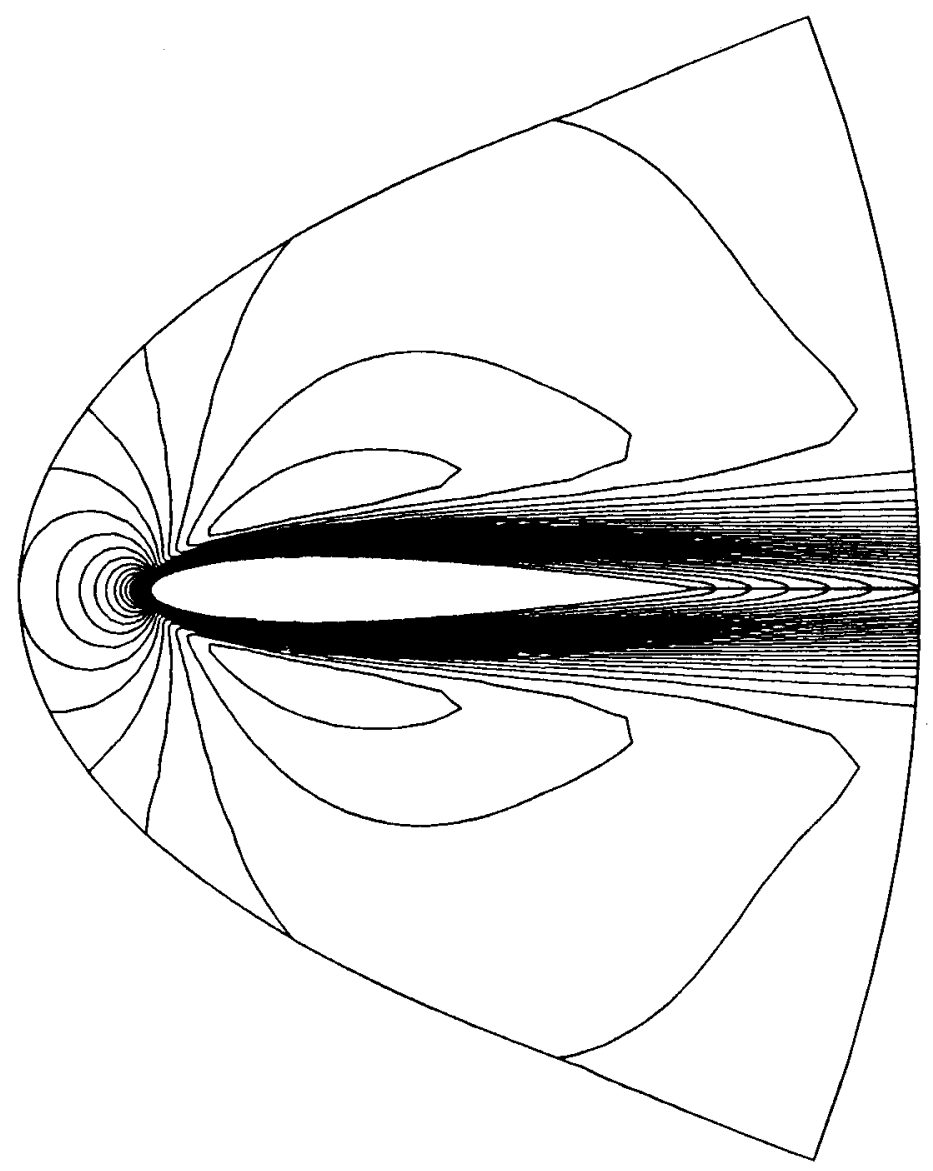

Figure 4.- MACH NUMBER CONTOURS For Viscous LAMINAR FLOW. 


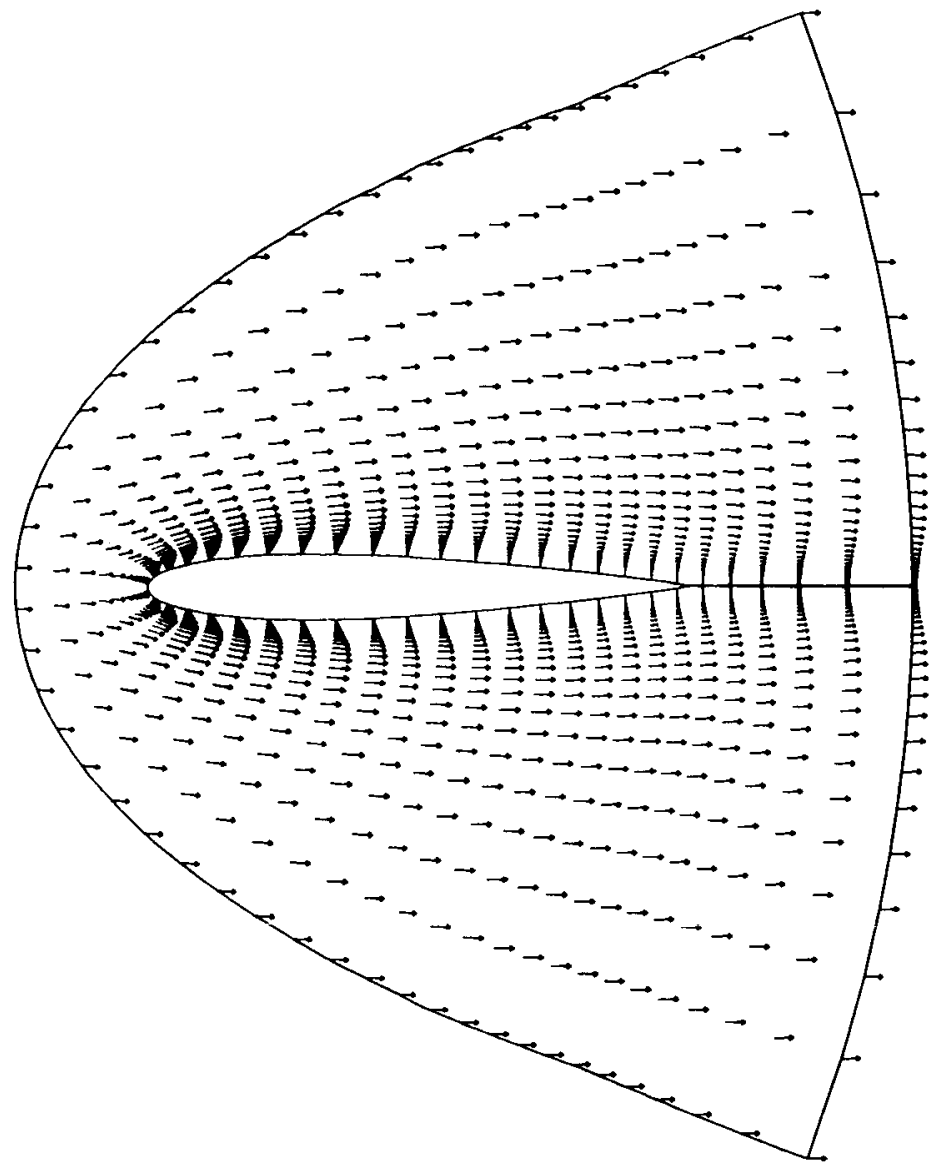

FIGURE 5. - VELOCITY VECTORS FOR VISCOUS LAMINAR FLOW.

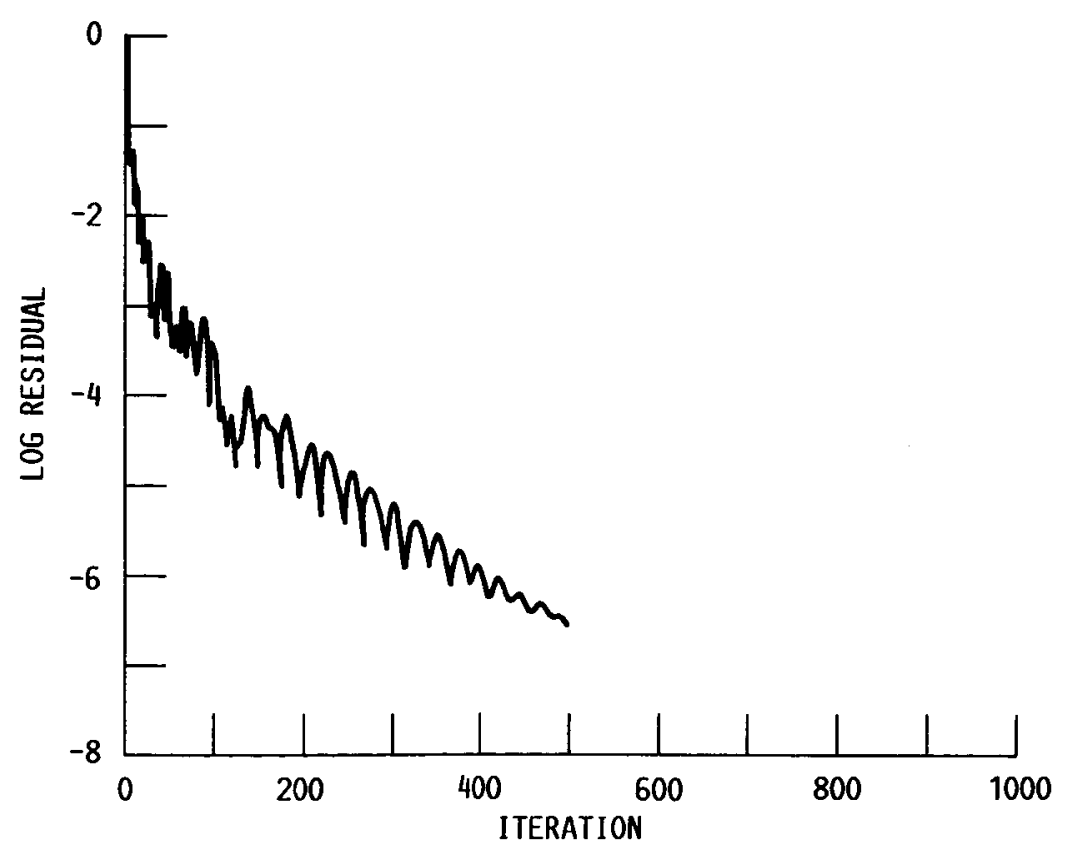

FigURE 6.- CONVERGENCE HISTORY FOR VISCOUS LAMINAR FLOW. 


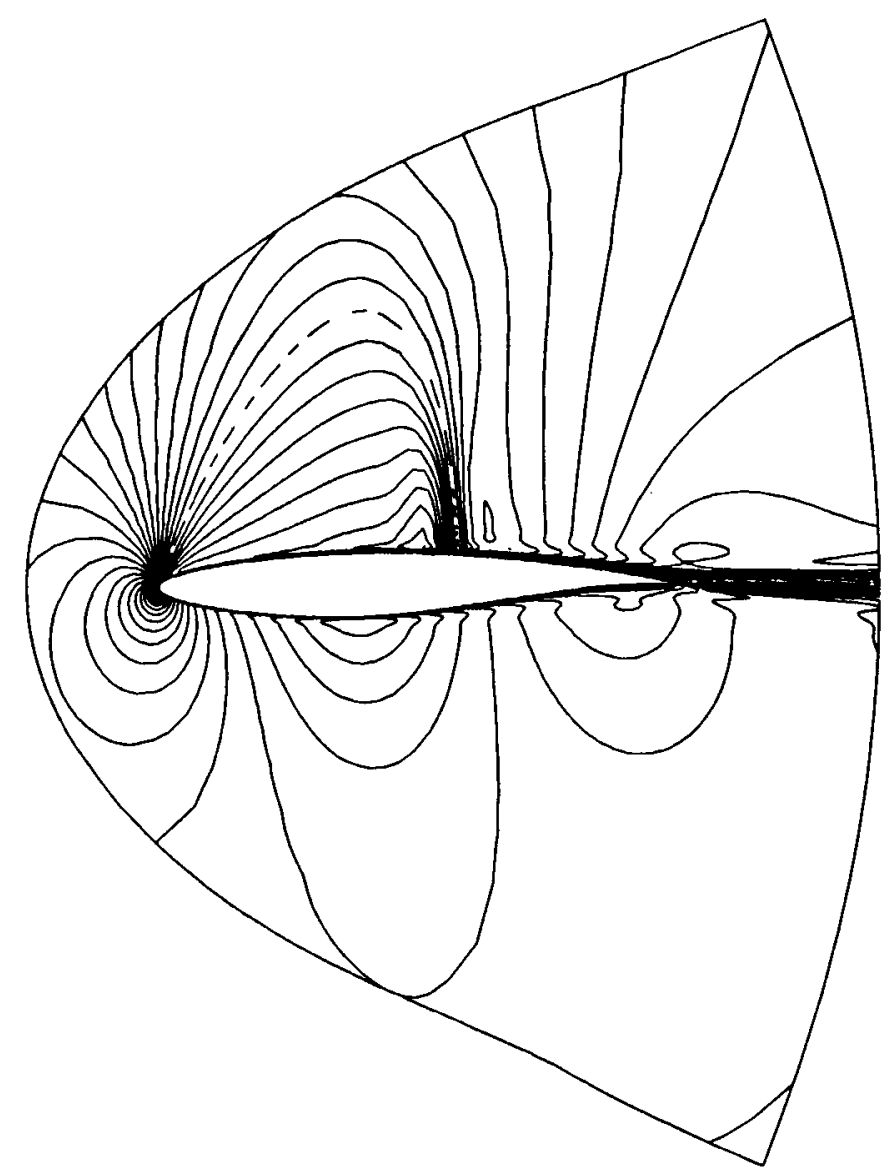

Figure 7.- Mach Number contours for Viscous turbulent flow.

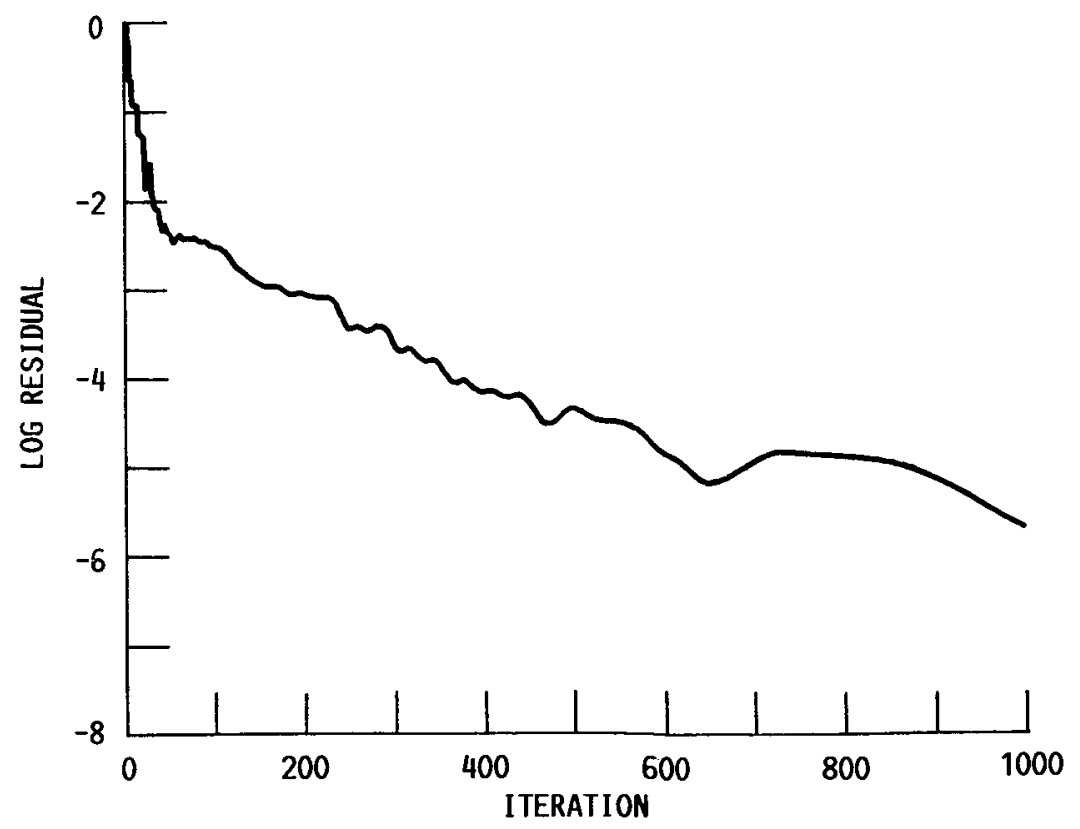

Figure 8. - CONVERGENCE HISTORY FOR VISCOUS TURBULENT FLOW. 


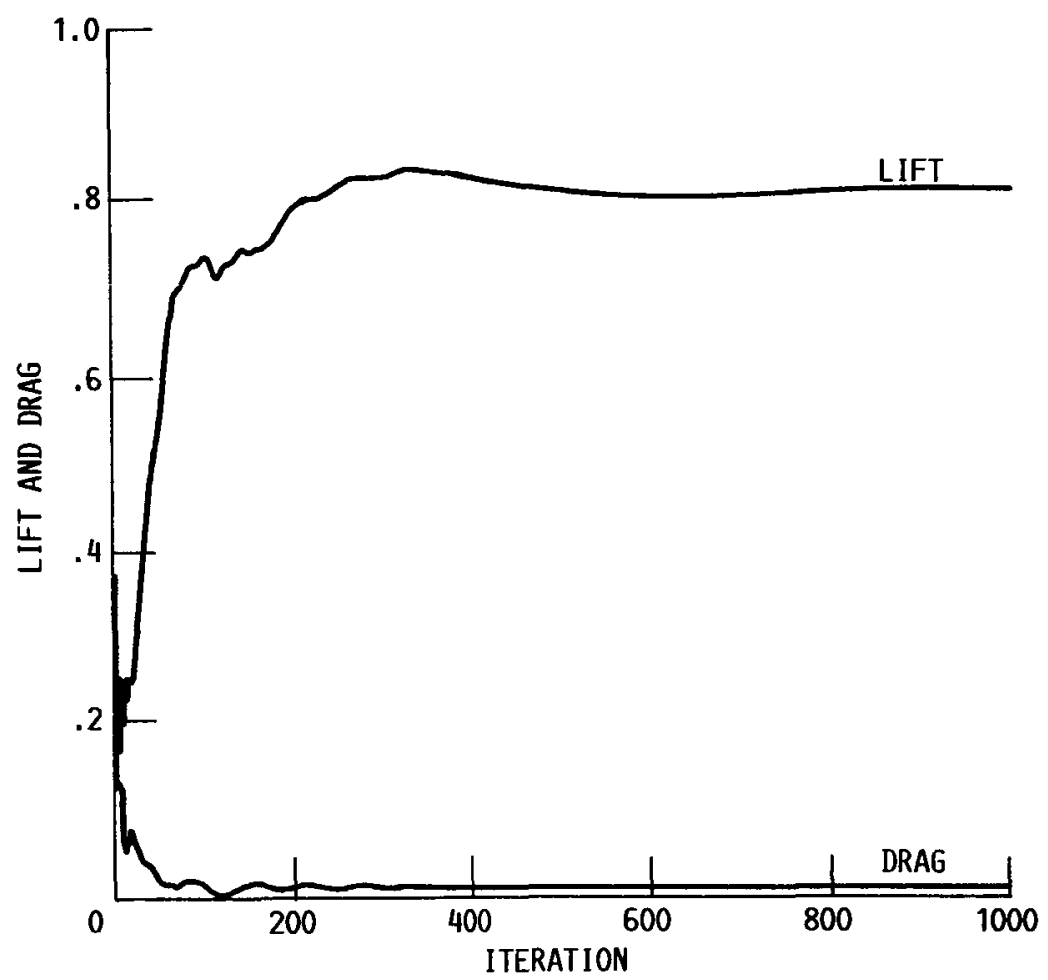

FIGURE 9. - LIFT AND DRAG HISTORIES FOR VISCOUS TURBULENT FLOW. 


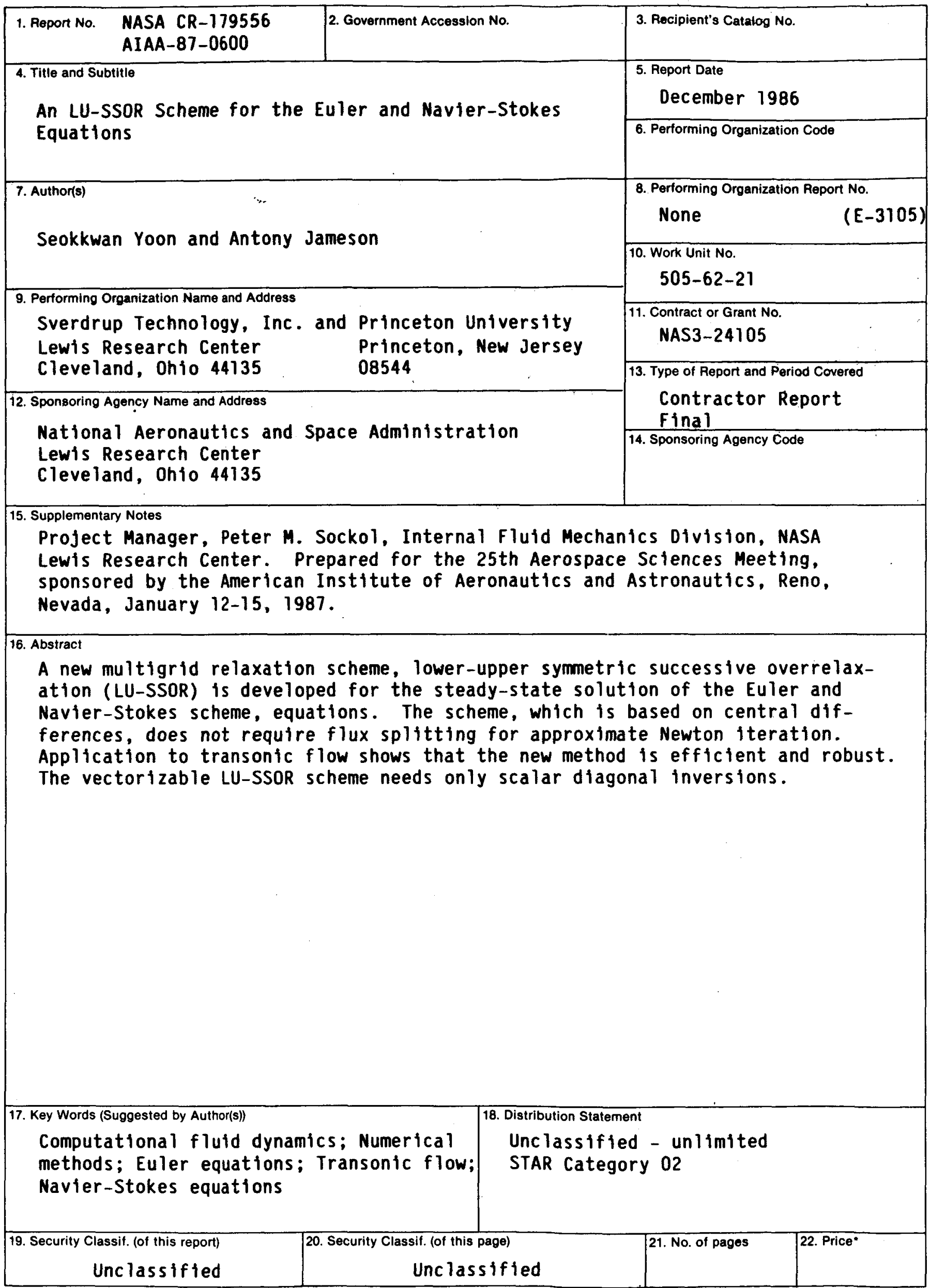

*For sale by the National Technical Information Service, Springfield, Virginia 22161 